\title{
In-Memory-Technologien für Unternehmensanwendungen
}

International zunehmender Wettbewerb, kürzere Produktlebenszyklen und eine hohe Marktdynamik erfordern die flexible und effiziente Verwendung von IT-Systemen innerhalb von Unternehmen. Mitunter müssen erfolgskritische Operationen wie Bestandsanalysen und Bedarfsvorhersagen über Nacht durchgeführt und bis zum nächsten Morgen abgeschlossen sein. Gleichzeitig fallen durch zunehmende Automatisierung immer größer werdende neue Datenmengen an, welche mit bereits vorhandenen Datenbeständen verknüpft und verarbeitet werden müssen. ${ }^{1}$

Zur zeitnahen Bearbeitung und Auswertung von großen Datenbeständen sind Unternehmen auf die kontinuierliche Weiterentwicklung von Informations- und Kommunikationstechnologien angewiesen. In diesem Zusammenhang spielt von technischer Seite aus die Entwicklung von sog. „In-MemoryTechnologien“ eine große Rolle. Durch die Verwendung von In-MemoryTechnologien können Daten im Schnitt 10- bis 100-mal schneller verarbeitet werden als mit herkömmlichen Mitteln (vgl. Loos et al. 2011).

Der Unterschied zur „klassischen“ Datenverarbeitung besteht darin, dass mit In-Memory-Technologien die Daten nicht von einem Sekundärspeicher (z.B. Festplatten) abgerufen und zur weiteren Bearbeitung in den Arbeitsspeicher (Primärspeicher) geladen, sondern sämtliche Daten zur Bearbeitung im Arbeitsspeicher des Computers verbleiben. Da keine Input-Output-Zugriffe auf den Sekundärspeicher mehr nötig sind, kann der Datenzugriff deutlich schneller erfolgen (vgl. Plattner/Zeier 2011). Gleichwohl es sich bei Arbeitsspeicher um sog. „nicht-persistenten“ Speicher handelt, d.h. dass Daten bei Systemabstürzen nicht mehr verfügbar sind, so existieren verschiedene Methoden, um die fehlende Persistenz des Arbeitsspeichers auszugleichen.

Die Idee Daten direkt im Arbeitsspeicher vorzuhalten und dort zu bearbeiten entstand bereits in den 90er Jahren. In den Folgejahren entstanden weitere, auf In-Memory-Technologien aufbauende Produkte von diversen Herstellern. Weiteren Schub erhielt diese Entwicklung aufgrund fallender Arbeitsspeicherpreise, die die Nutzung des Arbeitsspeichers als primären Speicherort für Daten zunehmend attraktiver machte. Voll ausschöpfen können In-Memory-Technologien ihr Potential jedoch erst durch die Entwicklung von 64-Bit-Prozessoren. Bei klassischen 32-BitComputersystemen liegt systembedingt eine Arbeitsspeicherobergrenze von 4 GigaByte vor - die Verwendung von größeren Datenmengen im Arbeitsspeicher ist ohne erneutes Lachladen somit nicht möglich. Erst durch die Entwicklung von 64-Bit-Prozessoren, welche Arbeitsspeichergrößeren von theoretisch bis zu 16 Exabyte (entspricht 16 Milliarden Gigabyte) erlauben,

\footnotetext{
${ }^{1}$ Für die Herausforderungen und Lösungen bei der Verarbeitung von großen Datenbeständen wird in letzter Zeit häufig der Begriff „Big Data“ verwendet.
} 
können In-Memory-Technologien nun auch zur Bearbeitung großer Datenbestände direkt im Arbeitsspeicher genutzt werden.

Meist geht die Nutzung von In-Memory-Technologien jedoch meist in Kombination mit der Verwendung weiterer Technologien und Verfahren einher. So führte zusätzlich zur Entwicklung der 64-Bit-Architektur insbesondere das Aufkommen von neueren Multi-Core-Prozessoren zu weiteren Effizienzsteigerungen. Diese Klasse von Prozessoren verfügt über mehrere vollständige Hauptprozessoren auf einem Chip und ermöglicht somit die simultane Auswertung mehrerer Prozesse.

Eine Übersicht über verschiedene, in Zusammenhang mit In-MemoryDatenmanagement verwendete Technologien und Anwendungsbereich zeigt Abbildung 1.

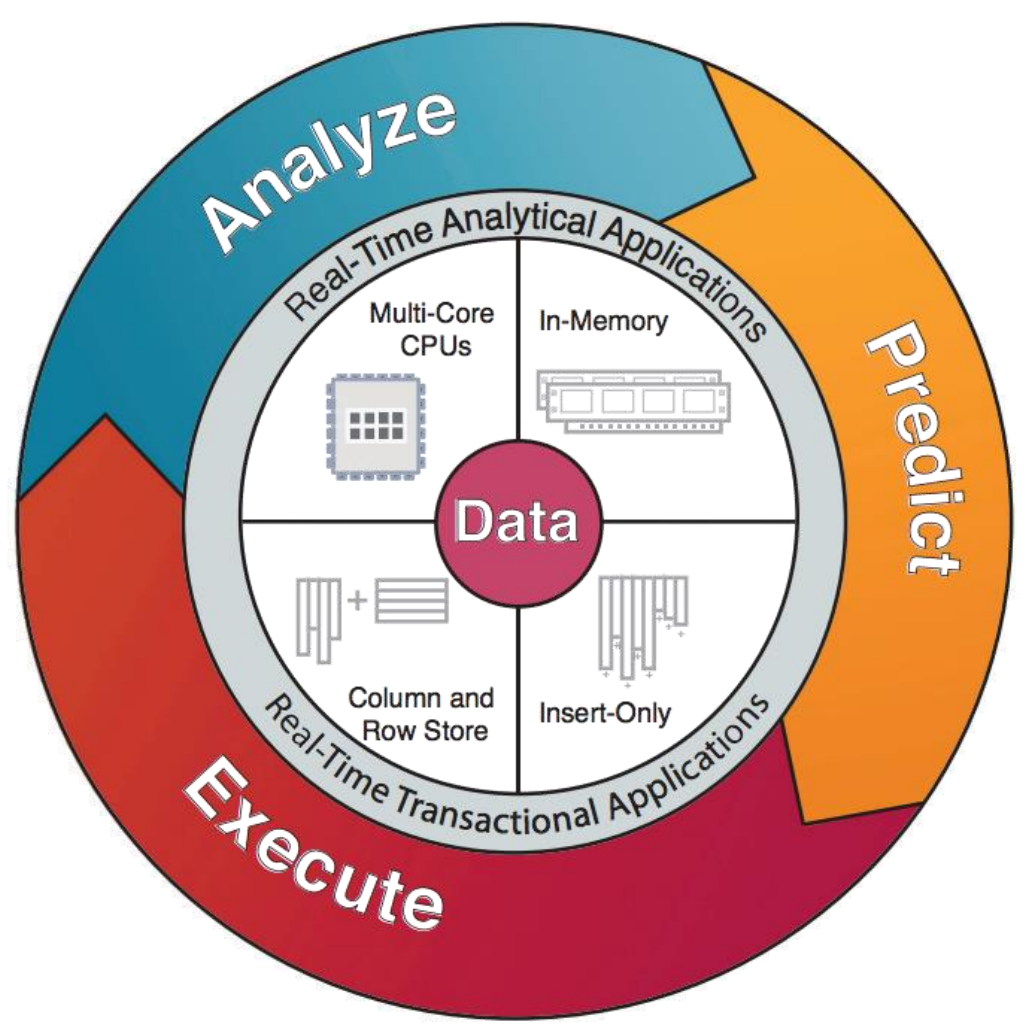

Abbildung 1: Übersicht über In-Memory-Technologien (Plattner/Zeier 2011).

Mit In-Memory-Technologien lassen sich große Datenmengen in kürzerer Zeit analysieren und entsprechend aufbereiten. Aufgrund der Geschwindigkeitsvorteile von In-Memory-Technologien finden solche Systeme besonders bei zeitkritischen operationalen Prozessen Anwendung. Insgesamt können sich für Unternehmen folgende Vorteile ergeben:

1. Schnellere Verfügbarkeit von Daten: Durch schnellere Berechnungszeiten können einzelnen Prozesse in einem Bruchteil der Zeit, mitunter sogar nahezu in Echtzeit, durchgeführt werden. Dies erlaubt bspw. die schnellere 
Reaktion auf Kundenanfragen oder aber den Zugriff auf Live-Zugriff auf Unternehmensdaten während Management-Sitzungen.

2. Vereinfachte Datenanalyse: Mit In-Memory-Anwendungen lassen sich strukturierte und unstrukturierte Daten aus verschiedenen Einzelsystemen einfacher zusammen analysieren, ohne dass zuvor eine Aggregation vorgenommen werden muss.

3. Steigerung der Datenqualität: Durch den zuvor genannten Direktzugriff auf Unternehmensdaten, liegen den Entscheidern nicht nur aktuellere Daten vor im gleichen Zuge entfallen auch mögliche Übertragungsfehler. Weiterhin lässt sich die Datenqualität auch dadurch steigern, dass für gewisse Berechnungen auf Stichproben verzichtet und nun der gesamte Datenbestand zur Berechnung herangezogen werden kann. Hinzu kommt, dass durch InMemory-Technologien auch bessere Berechnungsverfahre verwendet werden können, die jedoch aufgrund ihrer Berechnungskomplexität bislang nicht eingesetzt werden konnten.

4. Größere Datenbasis als Entscheidungsgrundlage: Durch schnellere Berechnungsdauern haben Unternehmen die Möglichkeit deutlich mehr Berechnungen innerhalb eines gewissen Zeitraums durchzuführen. Als Beispiel sind hier datenintensiven Szenarioanalysen, etwa zur Abschätzung von externen Marktparameter auf den Absatz der eigenen Produkte) zu nennen.

Somit können die Vorteile von In-Memory-Technologien grundsätzlich für alle Funktionen und Einheiten eines Unternehmens von Interesse sein. Speziell im Controlling unterstützt die höhere Flexibilität, die sich durch schnellere Auswertungsmöglichkeiten sowie die vereinfachte Datenanalyse ergibt, die Entwicklung und die Integration neuer Kennzahlen. Durch die verbesserte Datenqualität ergibt eine bessere Entscheidungsgrundlage für kurz- und langfristige strategische Planungen, was sich wiederum positiv auf den Unternehmenserfolg auswirken kann.

Aus Unternehmenssicht ist zu prüfen, inwieweit die Notwendigkeit für den Einsatz von In-Memory-Technologien besteht, bzw. inwieweit diese Technologien Veränderungen von Unternehmensprozessen ermöglichen können. Generell ist die Zweckmäßigkeit von In-Memory-Technologien erheblich von der Anzahl an zeitkritischen Operationen und Prozessen abhängig. Bei nicht zeitkritischen Anwendungen, bei denen bestehende ITSysteme ihre Leistungsgrenzen bislang nicht annähernd ausschöpfen, mag demnach eine Umstellung auf In-Memory-Technologien aufgrund der damit verbunden Schwierigkeiten und ggf. einhergehender Kosten nur bedingt attraktiv erscheinen. So werden bspw. retrospektive Datenauswertungen für monatliche Berichtserstattungen möglicherweise nur geringen Nutzen aus dieser Technologie ziehen können (vgl. Gartner 2011a).

Da die Auswertung großer Datenbestände über In-Memory 64-Bit-basierte Computersysteme voraussetzt, erfordert die Umstellung für Unternehmen mit bestehender 32-Bit-basierter Hard- und Softwarearchitektur zunächst 
möglicherweise gewisse Investitionen. Durch Nutzung von In-MemoryAnwendungen per "Software as a Service" (SaaS) können solche Investitionen jedoch ggf. reduziert werden. In diesem Fall betreibt der ITAnbieter der SaaS-Dienstleistung die jeweilige Software und die ITInfrastruktur. Allerdings müssen hierzu sämtliche zu analysierende Daten an den SaaS-Anbieter freigegeben werden. Besonders bei sensiblen Unternehmensdaten ist hierbei der sorgfältige Umgang des IT-Dienstleister mit den Daten sicherzustellen.

Weiterhin mangelt es Unternehmen an konkreten Business-Cases und verlässlichen Vorhersagen in Bezug auf die Umstellung auf In-MemoryTechnologien sowie auf die dadurch zu erzielenden Effizienzgewinne.

Aus technischer Perspektive ist In-Memory-Datenmanagement jedoch die logische Konsequenz technologischen Fortschritts. Die Entwicklung von 64Bit-Prozessoren und das steigende Angebot an kostengünstigem Arbeitsspeicher waren der Popularität dieser Technologie stark zuträchtig. Diese Technologien haben das Potential, weitgehende Veränderungen bestehender Business-Intelligence- und Data-Warehouse-Lösungen hervorzurufen. Das Marktforschungsinstitut Gartner prognostiziert In-MemoryTechnology als eines der zehn wichtigsten strategischen Technologien im Jahr 2012 (vgl. Gartner 2011b). Eine größere Anzahl an verlässlichen empirischen Daten und konkreten Business-Cases erscheint notwendig, um die in In-Memory-Technologien gesetzten Hoffnungen mit Fakten zu untermauern und weitere Akzeptanz auf Seiten der Anwender zu schaffen.

\section{Literaturquellen:}

Gartner 2011a: In-Memory Analytics: Leveraging Emerging Technologies for Business Intelligence, Betrachtet am 30.05.2012, http://ibmtelconewsletter.files.wordpress.com/2010/05/ar_inmemory_analytics _leveraging_emerging_technologies_for_business_intelligence.pdf.

Gartner 2011b: Gartner Identifies the Top 10 Strategic Technologies for 2012, Betrachtet am 30.05.2012, http://www.gartner.com/it/page.jsp?id=1826214.

Loos, P./Lechtenbörger, J./Vossen, G./Zeier, A./Krüger, J./Müller, J./Lehner, W./Kossmann, D./Fabian, B./Günther, O./Winter, R.: In-Memory-Datenmanagement in betrieblichen Anwendungssystemen, in: Wirtschaftsinformatik 53. Jg. (2011), Heft 6, S. 383-390.

Plattner, H./ Zeier, A.: In-Memory Data Management - An Inflection Point for Enterprise Applications, Berlin/Heidelberg 2011. 